\title{
Vessel sealing system for video-assisted lung resection for cancer reduces chylothorax and bleeding
}

\author{
Masahiro Miyajima, Ryunosuke Maki, Makoto Tada, Kodai Tsuruta, Yuki Takahashi, Wataru Arai, \\ Atsushi Watanabe \\ Department of Thoracic Surgery, Sapporo Medical University School of Medicine, Chuo-ku, Sapporo, Hokkaido, Japan \\ Contributions: (I) Conception and design: M Miyajima; (II) Administrative support: A Watanabe; (III) Provision of study materials or patients: M \\ Miyajima; (IV) Collection and assembly of data: M Miyajima; (V) Data analysis and interpretation: M Miyajima; (VI) Manuscript writing: All authors; \\ (VII) Final approval of manuscript: All authors. \\ Correspondence to: Masahiro Miyajima, MD. Department of Thoracic Surgery, Sapporo Medical University School of Medicine, Sapporo, South 1, \\ West 16, Chuo-ku, Sapporo, Hokkaido 060-8543, Japan. Email: miyajima@sapmed.ac.jp.
}

Background: The objective of this study was to analyze the efficacy of the LigaSure ${ }^{\mathrm{TM}}$ vessel sealing system for lung cancer resection with node dissection, as this has not been sufficiently evaluated.

Methods: From 2004 to 2018, 948 patients underwent anatomical pulmonary resection with node dissection for non-small cell lung carcinoma (NSCLC) via the video-assisted thoracoscopic surgery (VATS) approach. Medical records of these patients were reviewed retrospectively. Univariate and multivariate analyses were conducted to determine the risk factors for chylothorax and blood loss.

Results: Of the 948 patients, 318 (33.5\%) who underwent anatomical lung resection with node dissection by conventional methods without vessel sealing system and 630 (66.5\%) who underwent lung resection with node dissection with the vessel sealing system were included. The median intraoperative blood loss was $100 \mathrm{~mL}$. Postoperative chylothorax occurred in $9(2.8 \%)$ patients in the conventional method group with $2(0.3 \%)$ patients in the vessel sealing system group $(\mathrm{P}=0.001)$. Patients in the vessel sealing group who developed chylothorax were cured by conservative treatment. Univariate and multivariate analyses identified male sex [odds ratio (OR) 2.053; 95\% confidence interval (CI): 1.494-2.820; $\mathrm{P}<0.001$ ] and the use of vessel sealing system (OR 0.342; 95\% CI: 0.256-0.457; $\mathrm{P}<0.001$ ) as independent predictors of intraoperative blood loss. The univariate and multivariate analyses identified the use of the vessel sealing system (OR 0.108; 95\% CI: $0.023-0.504 ; \mathrm{P}=0.005)$ as an independent predictor of chylothorax incidence.

Conclusions: Vessel sealing system for lung cancer resection could decrease chest tube duration, amount of intraoperative bleeding, and incidence of chylothorax in patients who undergo lung resection with node dissection.

Keywords: Video-assisted thoracoscopic surgery (VATS); lung cancer; vessel sealing system; chylothorax

Submitted Jan 26, 2021. Accepted for publication Apr 23, 2021.

doi: $10.21037 /$ jtd-21-169

View this article at: http://dx.doi.org/10.21037/jtd-21-169

\section{Introduction}

The LigaSure ${ }^{\mathrm{TM}}$ vessel sealing system (VSS) (Valleylab, Boulder, CO, USA) is an electrosurgical device used to seal tissue against conventional clips and ligatures. The VSS fuses the vessel walls by applying the appropriate pressure and bipolar energy to the tissue. It melts the elastin and collagen of the vessel walls, and fuses them, obliterating the lumen. It has been used in thoracic, urological, abdominal, gynecological, and laparoscopic surgeries (1-6). Clinical results of the VSS have demonstrated its safety and effectiveness and capability to shorten operating times for surgeries, including abdominal surgery and hepatectomy. However, English literature reveals little studies using the 
VSS for the lung cancer resection. Thus, in this study, we aimed to evaluate the effects of the VSS on acute surgical outcomes when used for lung cancer resection with node dissection.

We present the following article in accordance with the STROBE reporting checklist (available at http://dx.doi. org/10.21037/jtd-21-169).

\section{Methods}

\section{Subjects and study design}

The study was conducted in accordance with the Declaration of Helsinki (as revised in 2013). The institutional review board approved the study protocol (Sapporo Medical University IRB NO. 322-310) and waived the need for written informed consent. From 2004 to 2018, a total of 948 patients underwent anatomical pulmonary resection with node dissection for non-small cell lung carcinoma (NSCLC) via the video-assisted thoracoscopic surgery (VATS) approach. This study was a retrospective analysis of patient data. Medical records of these patients were reviewed (Table 1). All these surgeries were performed by the same group of surgeons. Patients that required conversion to open thoracotomy or that had undergone either preoperative chemotherapy or radiotherapy were excluded from this study to simplify the investigation of VSS effectiveness in general cases. Patients who underwent surgery by partial resection were excluded from this study because the VSS is never or rarely used in this surgery.

\section{Operative procedure}

At our institution, VATS is usually performed via one utility incision $(30-40 \mathrm{~mm})$ at the level of the fourth intercostal space and two ports at the sixth and seventh intercostal space. The surgeon stands on the ventral side of the patient, whereas the scopist and the assistant are located on the frontal and opposite sides, respectively. In VATS anatomical lung resection surgery, the pulmonary artery branch was doubly ligated with 2-0 silk sutures or surgical clips and then divided (conventional method). In the VSS group, we ligated the proximal part of the pulmonary artery branch with a 2-0 silk suture and then divided the vessel with the VSS. After anatomic pulmonary resection, mediastinal lymph node dissection (MLND) is routinely performed. In the right side, mediastinal lymph node stations $2 \mathrm{R \# ,} \mathrm{4R \# ,}$ 7\#, \#8 and 9\# were dissected. For the left side, mediastinal lymph node stations 4L\#, 5\#, 6\#, 7\#, 8\# and 9\# dissection were dissected proposed by International Association for the Study of Lung Cancer (IASLC) guideline (7). In contrast, selective MLND was performed in patients who underwent segmentectomy. Staging was performed according to the Lung Cancer Staging Manual (7th edition) by the American Joint Committee on Cancer. Regarding MLND, initially, we used electrocautery and clips to divide the blood and lymphatic vessels (conventional method); our current technique relies on the VSS, which is quick and leads to less lymphorrhea (8). Chest tube was removed when the total discharge volume was not more than $5 \mathrm{mg} / \mathrm{kg} /$ day, and apparent air leakage nor chylothorax was not detected. Chylothorax is diagnosed based on the presence of chyle in the pleural fluid, triglyceride $>110 \mathrm{mg} / \mathrm{dL}$, or the presence of chylomicron, generally diagnosed by the sight of milky discharge after oral intake. A low-fat diet and pleurodesis were performed to treat chylothorax patients. In case chylothorax had not been cured in about 7 days and/ or the daily amount of chylous drainage fluid $>500 \mathrm{~mL}$ with this conservative treatment, surgical intervention, which is the VATS thoracic duct ligation, was considered.

\section{Statistical analysis}

Univariate logistic regression analysis was performed to first assess the association between the incidence of chylothorax, blood loss (median surgical blood loss of $100 \mathrm{~mL}$ or more) and the following patient characteristics: sex, age, body mass index (BMI), VSS usage, comorbidities (hypertension, diabetes mellitus), preoperative steroid use, lesion side, and resected lobe. The risk factors to be include in the multivariate analysis were selected considering the results of the univariate analyses, statistical independence and clinical importance. A multivariate logistic regression analysis with backward elimination was performed to identify independent predictors of chylothorax and intraoperative blood loss. It was also used to estimate the respective odds ratios (ORs) and $95 \%$ confidence intervals (CIs) of the various predictive factors. With regards to descriptive statistics, continuous variables were described using means or medians (interquartile ranges), while categorical variables were described using frequencies and percentages. All statistical analyses were performed using SPSS for Windows version 22.0 (SPSS, Inc., Chicago, IL, USA). Student's $t$-test or $\chi^{2} /$ Fisher's exact test as appropriate was used for analysis. All differences were considered significant at $\mathrm{P}<0.05$. 
Table 1 Patient demographics ( $\mathrm{n}=948)$

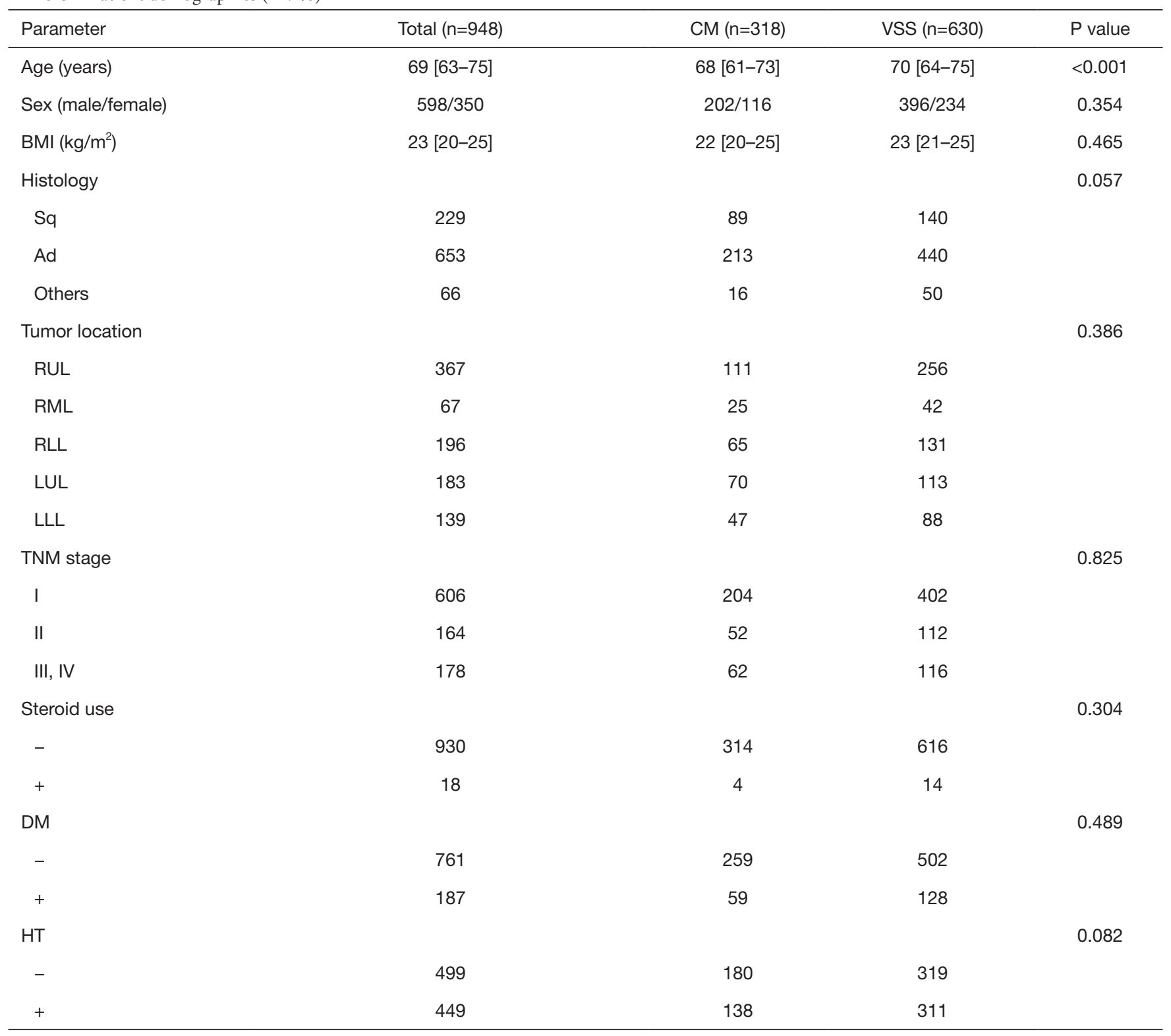

Ad, adenocarcinoma; BMI, body mass index; CM, conventional method; LLL, left lower lobectomy; LUL, left upper lobectomy; RLL, right lower lobectomy; RML, right middle lobectomy; RUL, right upper lobectomy; Sq, squamous cell carcinoma; VSS, vessel sealing system; DM, diabetes mellitus; HT, hypertension.

\section{Results}

The median patient age was 69 (range, 63-75) years. Tumor locations were the upper lobe $(\mathrm{n}=617,65.1 \%)$ and lower lobe $(\mathrm{n}=331,34.9 \%)$. We considered the right middle lobe as the upper lobe to analyze the relation of the tumor locations and the estimated blood loss (EBL) or chylothorax incidence in this paper. Histopathological evaluation revealed $653(68.9 \%)$ adenocarcinoma, 229
(24.2\%) squamous cell carcinoma, and $66(7.0 \%)$ other carcinoma; furthermore, 606 (63.9\%) patients were in stage I, $164(17.3 \%)$ patients in stage II, and 178 (18.8\%) patients in stage III or IV. Patient characteristics are shown in Table 1.

The median amount of intraoperative blood loss for all patients was $100 \mathrm{~mL}(50-250 \mathrm{~mL})$, while $11(1.2 \%)$ patients developed postoperative chylothorax.

The univariate and multivariate analyses identified male 
Table 2 Univariate and multivariate analyses for intraoperative blood loss ( $\mathrm{n}=948)$

\begin{tabular}{|c|c|c|c|c|c|c|c|}
\hline Factors & Categories & \multicolumn{3}{|c|}{ Univariate } & \multicolumn{3}{|c|}{ Multivariate } \\
\hline \multirow[t]{2}{*}{ Age (years) } & $<70$ & 1.000 & - & - & - & - & - \\
\hline & $\geq 70$ & 0.940 & $0.728-1.213$ & 0.634 & - & - & - \\
\hline BMI $\left(k g / m^{2}\right)$ & $<30$ & 1.000 & - & - & - & - & - \\
\hline \multirow[t]{2}{*}{ Sex } & Female & 1.000 & - & - & 1.000 & - & - \\
\hline & Male & 2.088 & $1.592-2.740$ & $<0.001$ & 2.053 & $1.494-2.820$ & $<0.001$ \\
\hline \multirow[t]{2}{*}{ VSS } & - & 1.000 & - & - & 1.000 & - & - \\
\hline & + & 0.349 & $0.263-0.462$ & $<0.001$ & 0.342 & $0.256-0.457$ & $<0.001$ \\
\hline \multirow[t]{2}{*}{ Resected lobe } & Lower lobe & 1.000 & - & - & - & - & - \\
\hline & Upper lobe & 0.941 & $0.724-1.237$ & 0.688 & - & - & - \\
\hline \multirow[t]{2}{*}{ Steroid use } & - & 1.000 & - & - & - & - & - \\
\hline & + & 0.844 & $0.322-2.212$ & 0.730 & - & - & - \\
\hline \multirow[t]{2}{*}{ DM } & - & 1.000 & - & - & - & - & - \\
\hline & + & 0.833 & $0.605-1.147$ & 0.262 & - & - & - \\
\hline \multirow[t]{2}{*}{$\mathrm{HT}$} & - & 1.000 & - & - & - & - & - \\
\hline & + & 0.952 & $0.738-1.230$ & 0.708 & - & - & - \\
\hline
\end{tabular}

BMI, body mass index; $\mathrm{Cl}$, confidence interval; OR, odds ratio; VSS, vessel sealing system; OP, operation; DM, diabetes mellitus; HT, hypertension.

sex (OR 2.088; 95\% CI: $1.592-2.740 ; \mathrm{P}<0.001$ and OR 2.053; 95\% CI: $1.494-2.820 ; \mathrm{P}<0.001$, respectively) and the use of VSS (OR 0.349; 95\% CI: $0.263-0.462 ; \mathrm{P}<0.001$ and OR 0.342 ; $95 \%$ CI: $0.256-0.457 ; \mathrm{P}<0.001$, respectively) as independent predictive factors of intraoperative blood loss (Table 2).

The univariate and multivariate analyses identified the use of VSS (OR 0.108; 95\% CI: 0.023-0.504; $\mathrm{P}=0.005$ and OR 0.108 ; 95\% CI: $0.023-0.504 ; \mathrm{P}=0.005$, respectively) as an independent predictive factor of chylothorax incidence. There was no correlation between lymph node metastases and chylothorax incidence $(\mathrm{P}=0.720)$ (Table 3). Hence, as additional analysis, we compared the operating time, amount of bleeding, intraoperative complications, and perioperative complications including chest tube duration between patients who had undergone lung resection with node dissection with the VSS (VSS group) and patients who had undergone the same surgery by conventional methods
(CM group) (Table 4). Surgery time, hospitalization and incidence of persistent air leaks (air leak lasts $>7$ days) did not differ significantly between two groups. Of the 11 patients with chylothorax, 10 were male. Their age ranged from 51 to 78 years. The initial procedure was lobectomy in 10 patients and bi-lobectomy in one patient. Lobectomy on the left side was performed in $4(36.4 \%)$ patients. Pathologic lymph node metastases were confirmed in three patients (all of them had p-N2 disease). Of these patients, three underwent redo surgery for the ligation of the injured thoracic duct. After reoperation, chylothorax was cured in all patients. The remaining eight patients continued a low-fat diet, and the condition was resolved. No patients underwent pleurodesis. Postoperative chylothorax was cured in all patients.

In these 11 patients, the chest tube was removed at a median of 6 days (4-7 days) after lung cancer surgery. There was no 30-day or in-hospital mortality in our study cohort. 
Table 3 Univariate and multivariate analyses for chylothorax ( $\mathrm{n}=948)$

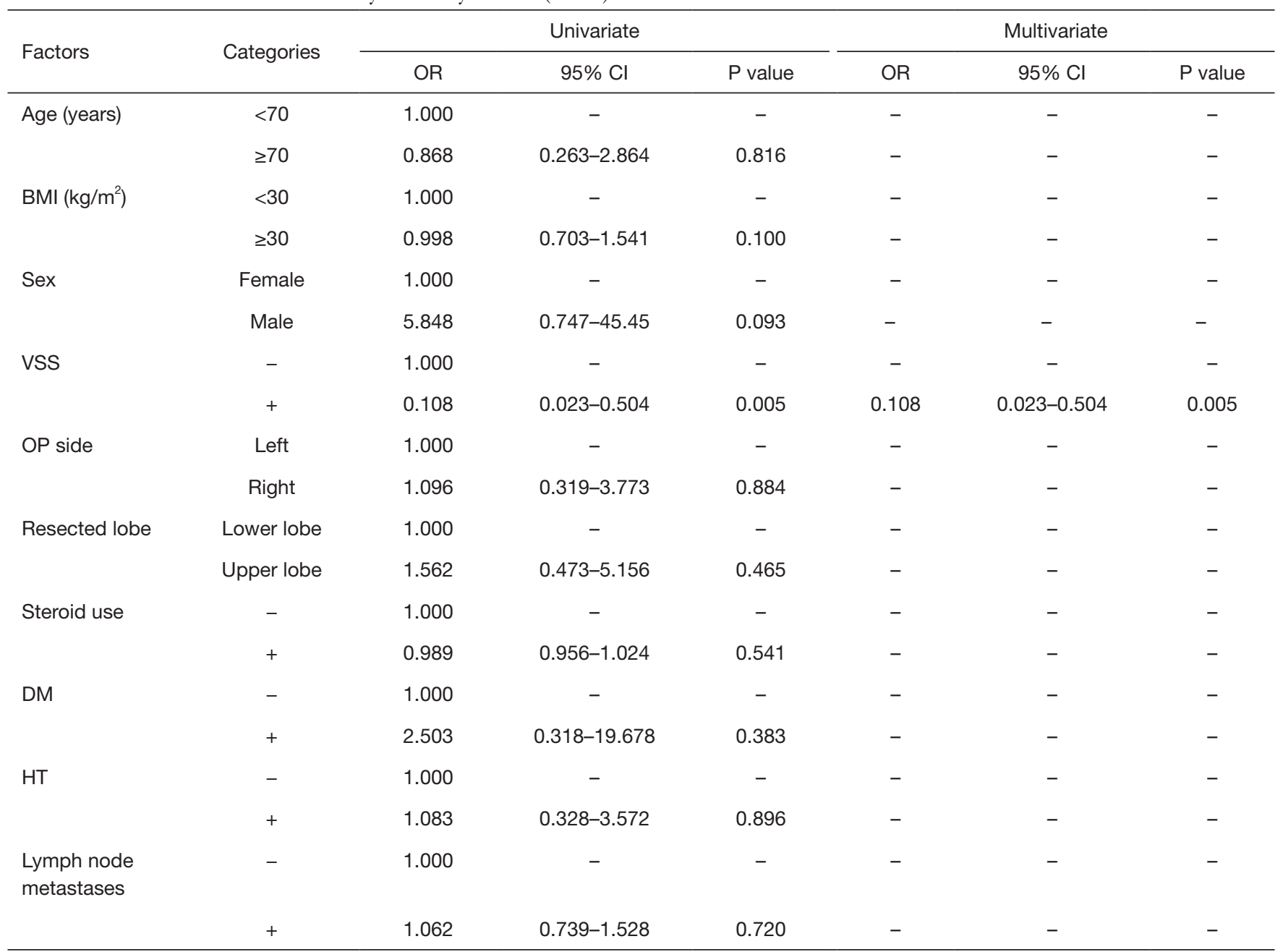

BMI, body mass index; $\mathrm{Cl}$, confidence interval; OR, odds ratio; VSS, vessel sealing system; OP, operation; DM, diabetes mellitus; HT, hypertension.

Table 4 Surgical outcomes

\begin{tabular}{|c|c|c|c|c|}
\hline Parameter & Total & $\mathrm{CM}$ & VSS & $P$ value \\
\hline Blood loss (mL) & $100[50-250]$ & 200 [70-380] & $100[30-200]$ & $<0.001$ \\
\hline Postoperative stay (days) & $10[7-14]$ & 9 [7-13] & $10[7-14]$ & 0.757 \\
\hline Chest tube duration (days) & $2[1-3]$ & $3[3-3]$ & $2[1-3]$ & 0.004 \\
\hline
\end{tabular}

CM, conventional method; VSS, vessel sealing system. 


\section{Discussion}

In this study, we evaluated the effectiveness of the VSS on acute surgical outcomes used for lung cancer resection with node dissection. Intraoperative blood loss and chest tube duration were significantly lower in the VSS group. There were no differences in the mean operating time and postoperative stay between the two groups.

These results were attributable to simple and reliable vascular and lymphatic closure and lymph node dissection by the VSS. Reduced intraoperative bleeding with VSS is due to the reduced need for extensive dissection and exposure of small pulmonary arteries and veins, which should be divided; bleeding from the distal site of the divided pulmonary vessels can also be easily controlled by the use of VSS. In the conventional group, we used electrocautery and clips to ligate the small vessels. Sufficient dissection is required when applying clips to control the thymic veins and bronchial arteries during lymph node dissection. Therefore, fragile vessels may get avulsed, leading to onerous bleeding. In addition, clips can easily slip off during manipulation. Mechanical staplers are also used to divide the pulmonary veins and the big pulmonary artery branches like superior truncus of the upper lobe and basilar artery of the lower lobe. While being useful, staplers require radical dissection and this increases the risk for small vessel injury, especially when inserting the jaws behind the branches. Moreover, the stapler cartridge is expensive in Japan (approximately \$250). In VSS, to divide the small pulmonary arteries, we put the proximal ligation and seal and cut the peripheral part with VSS.

We have previously described the double looping technique (How to clamp the main pulmonary artery during VATS lobectomy) and that the technique might reduce the need for emergent thoracotomy due to reduced intraoperative bleeding in VATS lung resection (9). Though the patients who underwent conversion to open thoracotomy were excluded in this study, the emergent thoracotomy rates in the CM and VSS groups were similar $(\mathrm{n}=4,1.3 \%$ vs. $\mathrm{n}=7,1.1 \% ; \mathrm{P}=0.842)$.

VATS became a viable treatment option for NSCLC with an equivalent oncological resection and equivalent or even superior oncological outcomes (10). In 2013, the Japanese Association of Thoracic Surgery reported that $70.8 \%$ of total surgeries for primary lung cancer were performed by VATS (11). VATS has been shown to be associated with fewer complications, shorter length of hospital stay, and less surgical morbidity $(12,13)$. However, some studies report no difference between VATS and thoracotomy (14).

In a previous study, we found that VATS could significantly reduce the prevalence of both minor and major complications (15). Similar findings were reported in other studies $(16,17)$.

In VATS surgery, the injury of the pulmonary arteries and the resulting massive bleeding has been regarded as the biggest obstacle. Different diathermy techniques have been developed to overcome the obstacle. In 1998, Kennedy reported the effectiveness of the LigaSure ${ }^{\mathrm{TM}}$ system for vessel sealing in animal model (18). The LigaSure ${ }^{\mathrm{TM}}$ system is reported as a unique bipolar system that can seal vessels up to $7 \mathrm{~mm}$ by application of precise pressure and thermal energy. This seal has been reported to have burst strength comparable with that of mechanical devices and ligatures in experimental studies (18).

In 2015, Martucci et al. reported the results of a pilot prospective study comparing the incidence of postoperative atrial fibrillation after lobectomy with nodal dissection by LigaSure ${ }^{\mathrm{TM}}$ system to those by electrocautery (19). In this report, no significant difference in atrial fibrillation onset rates was found between the two groups. LigaSure ${ }^{\mathrm{TM}}$ use was associated with reduction in mediastinal nodal dissection time $(\mathrm{P}=0.017)$ and fluid output duration $(\mathrm{P}=0.025)$; however, though there was no reduction in cumulative chest tube drainage volume $(\mathrm{P}=0.52)$ and chest tube duration $(\mathrm{P}=0.32)$ (19).

When pulmonary resection with MLND is performed for primary lung cancer, chylothorax is sometimes experienced as a postoperative complication. It usually prolongs hospitalization, and occasionally requires reoperation. During MLND, iatrogenic damage to the branches of the thoracic duct may cause chylothorax $(20,21)$. Some authors have suggested that the increased incidence of chylothorax following lung cancer surgery is caused by more aggressive MLND (22-24). MLND should be performed more meticulously due to the advancement of imaging systems, developing endoscopic instruments, and increased use of VATS. Nevertheless, no detailed studies have been conducted on the incidence of chylothorax after VATS lung cancer resection with node dissection. In a previous study, we reported a chylothorax rate of $1.4 \%$ after VATS MLND for lung cancer (15). The use of the VSS played a vital role in the reduction of the incidence rate of postoperative chylothorax. Liu et al. reported that 20 (2.6\%) of 776 patients with primary NSCLC who underwent VATS for pulmonary resection and MLND experienced chylothorax, which was treated conservatively; however, 5 of the 20 (25\%) patients 
subsequently required reoperation (24).

In this study, the incidence of postoperative chylothorax in the VSS group $(0.3 \%)$ was significantly lower than that in the CM group (2.8\%) $(\mathrm{P}=0.001)$. Moreover, all patients who developed chylothorax in the VSS method group were cured with conservative therapy. We experienced a great advantage by VSS use in node dissection: VSS use reduced postoperative effusions and lymphatic fistulae. Toishi et al. recently reported that VSS application reduced intraoperative bleeding, surgeon stress, postoperative drainage output, and drainage persistence compared with non-use VSS (25).

There was no post-operative hospital stay advantage in the VSS group but rather increase by 1 day (9 vs. 10 days, $\mathrm{P}=0.757)$. It would be resulted from the situation that clear criteria for hospital discharge after surgery have not been set in this study. While the duration of hospital stay may seem long for lung resection, it is lower than the 15.5 days reported by the Japanese Hospital Intelligence Agency data base (2020) patients (26).

A cost analysis comparison between lung resection using conventional and VSS techniques revealed modest cost difference. In Japan, hospitals do not charge for use of operating rooms, and therefore, the operating time does not affect the cost. As we carefully selected patients for blood transfusion, there was no significant difference in blood transfusion between the groups (3.0\% and 1.9\%). Considering the cost analysis, to use the VSS about $\$ 220$ more cost is required. But it is not expensive considering the benefits for patients (less chyle leak and chest tube out earlier). We strongly believe the blood loss reduction of $100 \mathrm{~mL}$ can contribute to the precision and safety of our VATS lung cancer surgery.

This study had some limitations. Its retrospective nature and lack of randomization may have led to potential bias in patient selection. Prospective, randomized studies are necessary to further understand the effectiveness of the VSS for lung cancer resection with node dissection. Furthermore, the incidence of chylothorax after lung cancer surgery was quite low, so the total number of chylothorax patients is limited.

In conclusion, the chest tube duration, amount of bleeding, and incidence of chylothorax in patients who had undergone VATS lung resection with node dissection can be reduced when using the VSS compared with that when using conventional methods.

\section{Acknowledgments}

Funding: None.

\section{Footnote}

Reporting Checklist: The authors have completed the STROBE reporting checklist. Available at http://dx.doi. org/10.21037/jtd-21-169

Data Sharing Statement: Available at http://dx.doi. org/10.21037/jtd-21-169

Peer Review File: Available at http://dx.doi.org/10.21037/jtd21-169

Conflicts of Interest: All authors have completed the ICMJE uniform disclosure form (available at http://dx.doi. org/10.21037/jtd-21-169). The authors have no conflicts of interest to declare.

Ethical Statement: The authors are accountable for all aspects of the work in ensuring that questions related to the accuracy or integrity of any part of the work are appropriately investigated and resolved. The study was conducted in accordance with the Declaration of Helsinki (as revised in 2013). The institutional review board approved the study protocol (Sapporo Medical University IRB NO. 322-310) and waived the need for written informed consent.

Open Access Statement: This is an Open Access article distributed in accordance with the Creative Commons Attribution-NonCommercial-NoDerivs 4.0 International License (CC BY-NC-ND 4.0), which permits the noncommercial replication and distribution of the article with the strict proviso that no changes or edits are made and the original work is properly cited (including links to both the formal publication through the relevant DOI and the license). See: https://creativecommons.org/licenses/by-nc-nd/4.0/.

\section{References}

1. Metzelder ML, Kübler J, Petersen C, et al. Laparoscopic nephroureterectomy in children: a prospective study on Ligasure versus clip/ligation. Eur J Pediatr Surg 2006;16:241-4.

2. Shigemura N, Akashi A, Nakagiri T, et al. A new tissue-sealing technique using the Ligasure system for nonanatomical pulmonary resection: preliminary results of sutureless and stapleless thoracoscopic surgery. Ann Thorac Surg 2004;77:1415-8.

3. Dilek ON, Yilmaz S, Degirmenci B, et al. The use of a 
vessel sealing system in thyroid surgery. Acta Chir Belg 2005;105:369-72.

4. Palazzo FF, Francis DL, Clifton MA. Randomized clinical trial of Ligasure versus open haemorrhoidectomy. Br J Surg 2002;89:154-7.

5. Tamussino K, Afschar P, Reuss J, et al. Electrosurgical bipolar vessel sealing for radical abdominal hysterectomy. Gynecol Oncol 2005;96:320-2.

6. Saiura A, Yamamoto J, Koga R, et al. Usefulness of LigaSure for liver resection: analysis by randomized clinical trial. Am J Surg 2006;192:41-5.

7. Vallières E, Shepherd FA, Crowley J, et al. The IASLC Lung Cancer Staging Project: proposals regarding the relevance of TNM in the pathologic staging of small cell lung cancer in the forthcoming (seventh) edition of the TNM classification for lung cancer. J Thorac Oncol 2009;4:1049-59.

8. Watanabe A, Nakazawa J, Miyajima M, et al. Thoracoscopic mediastinal lymph node dissection for lung cancer. Semin Thorac Cardiovasc Surg 2012;24:68-73.

9. Watanabe A, Koyanagi T, Nakashima S, et al. How to clamp the main pulmonary artery during VATS lobectomy. Eur J Cardiothorac Surg 2007;31:129-31.

10. Yan TD, Black D, Bannon PG, et al. Systematic review and meta-analysis of randomized and nonrandomized trials on safety and efficacy of video-assisted thoracic surgery lobectomy for early-stage non-small-cell lung cancer. J Clin Oncol 2009;27:2553-62.

11. Committee for Scientific Affairs, The Japanese Association for Thoracic Surgery, Masuda M, Kuwano H, et al. Thoracic and cardiovascular surgery in Japan during 2013: Annual report by The Japanese Association for Thoracic Surgery. Gen Thorac Cardiovasc Surg 2015;63:670-701. Erratum in: Gen Thorac Cardiovasc Surg 2016 ;64:496500. Gen Thorac Cardiovasc Surg 2017;65:182-6.

12. Paul S, Sdrakyan A, Chiu Y, et al. Outcomes after lobectomy using thoracoscopic vs thoracotomy: a comparative effectiveness analysis utilizing the nationwide inpatient sample database. Eur J Cardiothorac Surg 2013;43:813-7.

13. Phillips JD, Merkow RP, Sherman KL, et al. Factors affecting selection of operative approach and subsequent short-term outcomes after anatomic resection for lung cancer. J Am Coll Surg 2012;215:206-15.

14. Boffa DJ, Dhamija A, Kosinski AS, et al. Fewer complications result from a video-assisted approach to anatomic resection of clinical stage I lung cancer. J Thorac Cardiovasc Surg 2014;148:637-43.

15. Watanabe A, Koyanagi T, Ohsawa H, et al. Systematic node dissection by VATS is not inferior to that through an open thoracotomy: a comparative clinicopathologic retrospective study. Surgery 2005;138:510-7.

16. Law TD, Boffa DJ, Detterbeck FC, et al. Lethality of cardiovascular events highlights the variable impact of complication type between thoracoscopic and open pulmonary lobectomies. Ann Thorac Surg 2014;97:993-9.

17. Papiashvilli M, Sasson L, Azzam S, et al. Video-assisted thoracic surgery lobectomy versus lobectomy by thoracotomy for lung cancer: pilot study. Innovations (Phila) 2013;8:6-11.

18. Kennedy JS, Stranahan PL, Taylor KD, et al. High-burststrength, feedback-controlled bipolar vessel sealing. Surg Endosc 1998;12:876-8.

19. Martucci N, Tracey M, La Rocca A, et al. A pilot prospective randomized, controlled trial comparing LigaSure $^{\mathrm{TM}}$ tissue fusion technology with the ForceTriad ${ }^{\mathrm{TM}}$ energy platform to the electrosurgical pencil on rates of atrial fibrillation after pulmonary lobectomy and mediastinal lymphadenectomy. Eur J Cardiothorac Surg 2015;47:e13-8.

20. Suemitsu R, Ondo K, Fukuyama S, et al. Late-periodonset chylothorax after a pulmonary resection for lung cancer: a case report. Ann Thorac Cardiovasc Surg 2007;13:345-8.

21. Shimizu K, Yoshida J, Nishimura M, et al. Treatment strategy for chylothorax after pulmonary resection and lymph node dissection for lung cancer. J Thorac Cardiovasc Surg 2002;124:499-502.

22. Cho HJ, Kim DK, Lee GD, et al. Chylothorax complicating pulmonary resection for lung cancer: effective management and pleurodesis. Ann Thorac Surg 2014;97:408-13.

23. Takuwa T, Yoshida J, Ono S, et al. Low-fat diet management strategy for chylothorax after pulmonary resection and lymph node dissection for primary lung cancer. J Thorac Cardiovasc Surg 2013;146:571-4.

24. Liu C, Pu Q, Guo C, et al. Non-grasping en bloc mediastinal lymph node dissection for video-assisted thoracoscopic lung cancer surgery. BMC Surg 2015;15:38.

25. Toishi M, Yoshida K, Agatsuma H, et al. Usefulness of vessel-sealing devices for $\leq 7 \mathrm{~mm}$ diameter vessels: a randomized controlled trial for human thoracoscopic 
lobectomy in primary lung cancer. Interact Cardiovasc Thorac Surg 2014;19:448-55.

26. Hospital Intelligence Agency. Diagnosis Procedure

Cite this article as: Miyajima M, Maki R, Tada M, Tsuruta K, Takahashi Y, Arai W, Watanabe A. Vessel sealing system for video-assisted lung resection for cancer reduces chylothorax and bleeding. J Thorac Dis 2021;13(6):3458-3466. doi: 10.21037/ jtd-21-169
Combination Statistics. Accessed November 2, 2020. Available online: https://hospia.jp/dpc 